\title{
A CASE OF AMYOPLASIA CONGENITA, WITH PATHOLOGICAL REPORT
}

\author{
BY
}

\author{
DOROTHY STOPFORD PRICE, M.B. \\ Physician to St. Ultan's Infant Hospital, Dublin. \\ Clinical Assistant (Children), Royal City of Dublin Hospital.
}

This communication presents a record of the investigations made in a case of amyoplasia congenita (arthrogryposis multiplex congenita, multiple congenital articular rigidity).

\section{Clinical report.}

A female infant, aged six weeks, was referred to St. Ultan's Hospital, Dublin, on April 14th, 1932, by Dr. O'Donoghue of Aunascaul, Co. Kerry. She was the eighth child of healthy parents, all the children being alive and well. There was no history of ill-health or deformity on either side of the family, nor of intermarriage. The birth was a normal vertex presentation, and the midwife noticed that there was little or no amniotic fluid. The condition at 6 weeks showed a well-nourished female child weighing $8 \frac{1}{2} \mathrm{lb}$. (Fig. 1 and 2 show her at $4 \frac{1}{2}$ months.)

The arms showed no voluntary movement at the shoulders or elbows. The elbows appeared thickened and spindle-shaped. The left elbow joint could be flexed with difficulty to a right angle, and there was only slight flexion in the right. The hands lay in a position of congenital club hand with ulnar deviation; the wrists were flexed and could not be fully extended; the finger joints were flexed with the fingers partially overlapping each other and slightly webbed. The fingers could be separated and extended with difficulty. The skin on the palmar surfaces was tight and contracted. The legs were everted and stiff. The eversion could be corrected, but correction was impeded by spasm. The thighs lay in a position of coxa vara, and sometimes in scissor position, with thighs everted. There was some thickening of the knee joints, but the knees could be flexed and extended passively. The spine was held straight and rigid. This was due to spasm and disappeared later. The chest was normal except that the neck muscles appeared weak.

X-ray examination by Dr. McDonogh showed bilateral dislocation of the hips. No other bony deformity was observed. The diminished muscle shadow showed in the X-ray of the upper limb (Fig. 3).

On the advice of Mr. Arthur Chance, the hands were put up in plaster casts, remoulded once a week to an improved position, and removed daily for massage and exercises. As the position of the hands improved the whole upper limb was put up in the same way, with increasing degrees of flexion at the elbow, and still with daily movements and massage. The trunk, shoulder, neck and leg muscles were also massaged.

After 3 months treatment, on the publication of Dr. Sheldon's case ${ }^{1}$, the faradic reaction of muscles was tested. Owing to difficulties due to the size and age of the patient, it was felt at the time that the furdings might be unreliable, and the subsequent dissection of the arm proved this to be the case. At this stage, at the age of $4 \frac{1}{2}$ months, the condition was as follows:-The child could raise both arms from the shoulders, apparently by lifting the scapulæ; there was some degree of rotatory movement of the arms; no voluntary flexion of the elbow joints; the hands had opened up, the fingers spread, and the wrists had assumed a normal position though they tended to relapse if the splints were left off for long. The child could 
hesitatingly reach up and grasp a pencil held horizontally above her. Her intelligence was remarkable. The legs had loosened out and she was beginning to kick. The head was not held up as well as is normal at $4 \frac{1}{2}$ months, but tended to fall forwards. The spasm causing rigidity of the spine had disappeared. The child developed a severe attack of enteritis at 5 months, terminating in an intussusception, and died on July 24th, 1932.

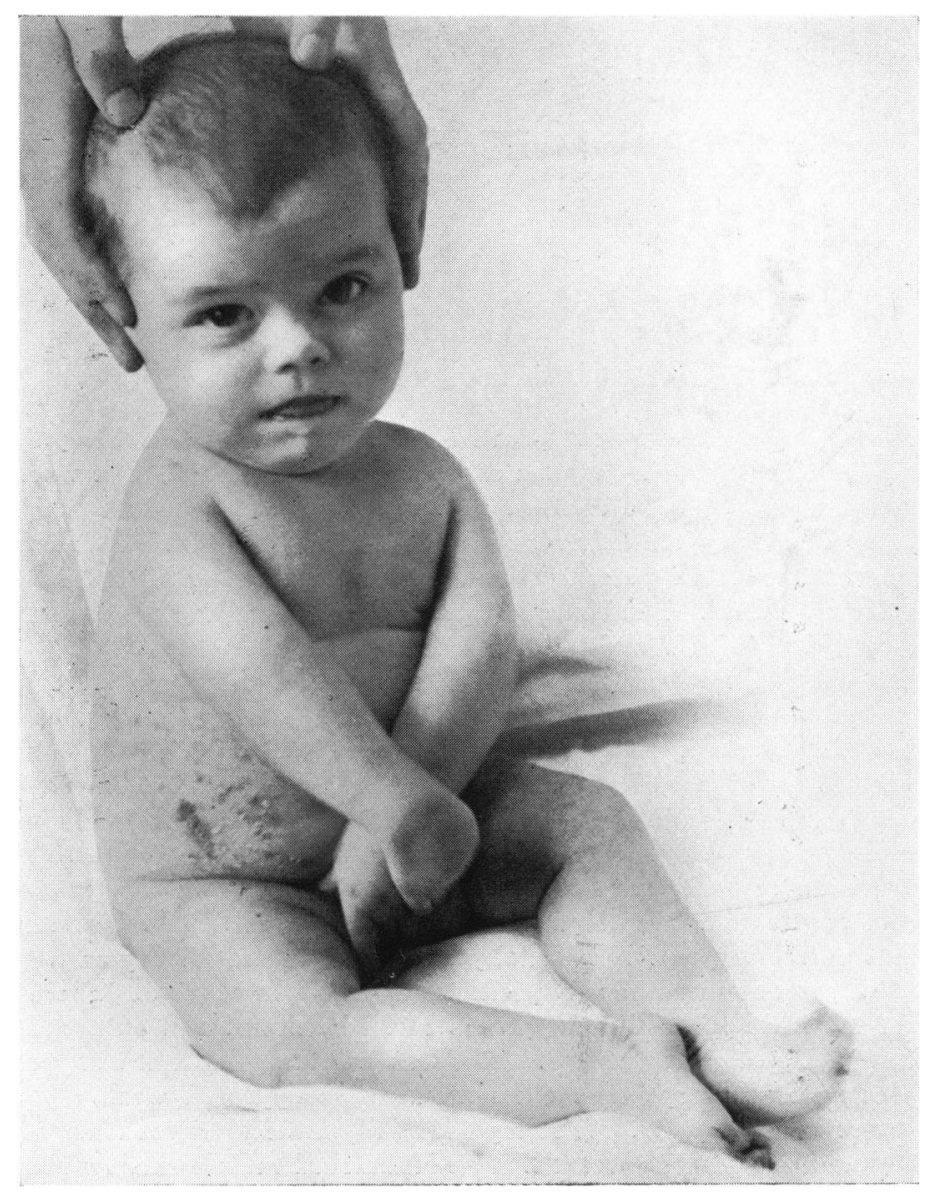

Fic. 1. Patient aged $4 \frac{1}{2}$ months.

Post mortem examination.

The body of a well-nourished infant, with the limbs in characteristic attitude, arms rotated in, elbows extended, wrists and fingers flexed. The skull was thin, the anterior fontanelle measured $2 \frac{1}{2}$ by 3 inches. The dura was adherent to the skull, but the brain was apparently normal. The pituitary was removed. From the abdomen the spleen, right suprarenal and right kidney were removed. The small intestine was generally rather congested, and showed an intussusception of the ileum into ascending colon, the entering layer being deeply congested. Portions of quadriceps femoris and glutæus maximus were removed. The right sterno-clavicular joint was disarticulated and the right upper limb removed. The sterno-mastoid muscles appeared well developed. The spinal cord was removed from 4 th cervical to 4th thoracic segments. The thymus gland was enlarged. 


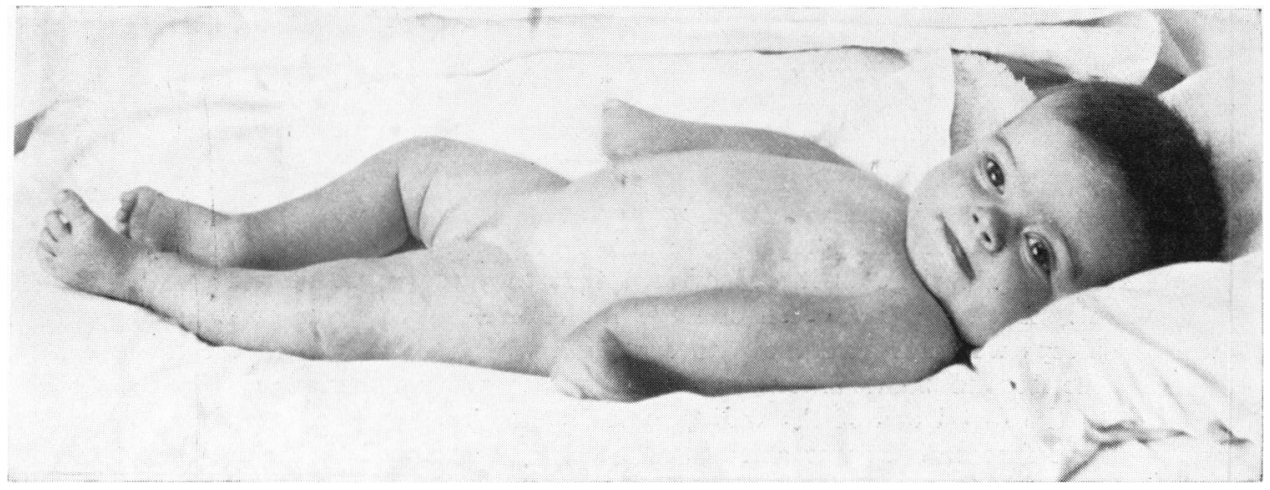

FIG. 2. Patient aged $4 \frac{1}{2}$ months.

FIt. 3. Skiagram showing congenital dislocation of hips and aiminished muscle shadow in upper limb. 
Report on dissection of elbow joint-(Dr. C. P. Martin and Mr. E. B. McEntee).-The skin was normal. The elbow joint was slightly flexed, the arm and forearm forming an angle of $\mathbf{1 5 0}$ degrees. It could be moved, with difficulty, through an angle of 15 degrees. The forearm was pronated, the wrist flexed and slightly adducted. The fingers were flexed and the thumb drawn across the palm.

On dissection the superficial fascia was abundant. It presented two well defined bands, one lying over the biceps the other over the brachioradialis. From their composition and attachments it was evident that they could not limit flexion of the elbow joint. Each was composed of loose fat covered by a layer of fascia. That over the biceps was attached above to the coracobrachialis, below to the tendon of biceps. The second band extended from the lateral supracondylar ridge of the humerus to the distal end of the radius, covering the brachioradialis and the lateral border of the forearm. It was smooth, rounded, and attached to the deep fascia on the volar and dorsal aspects by its fascial sheath.

1. Muscles connecting the aRM With the vertebral column.-The trapezius, latissimus dorsi, rhomboid major and rhomboid minor were normal; and the levator scapulæ was not available.

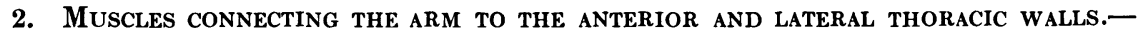
The pectoralis major, pectoralis minor, and serratus anterior were normal; and the subclavius not available.

3. Muscles of THE shoulder.-The deltoid was greatly reduced in size. The origin from the lower lip of the spine of the scapula was very small. Cleaning and definition of the muscle was made difficult by the presence of fibro-fatty material between the muscle bundles. The subscapularis, supraspinatus, infraspinatus, teres major, and teres minor were all normal.

4. MUSCLES OF THE ARM.-The coracobrachialis and triceps brachii were normal. The biceps brachii was covered by the fatty band mentioned above. When the band was dissected away the muscle was found to be very slender. The short head was tendinous and small, the long head constituting the greater bulk of the muscle. The brachialis was almost entirely replaced by fibro-fatty material. A small bundle of muscle fibres was present along the lateral and posterior part.

5. Muscles of the FOREARM.-In the superficial group of the volar antibrachial muscles, the pronator teres, flexor carpi radialis, palmaris longus, flexor carpi ulnaris, and the flexor digitorum sublimis were normal. In the deep group the flexor pollicis longus, flexor digitorum profundus and the pronator quadratus were normal.

In the superficial group of the dorsal antibrachial muscles the brachioradialis was reduced in size, especially in its upper portion; the extensor carpi radialis longus was reduced in size; the extensor carpi radialis brevis was absent or so small that it could not be seen with the naked eye; and the extensor digitorum communis, extensor digiti quinti proprius, extensor carpi ulnaris, and the anconæus were normal. In the deep group, the supinator was normal; abductor pollicis longus showed a radial head which was slender and fatty; the extensor pollicis brevis was very slender; and the extensor pollicis longus, and extensor indicis proprius were normal.

SyNOPSIS OF ABNORMAL MUSCles. 1. Absent:-extensor carpi radialis brevis. 2. Reduced in size:-biceps, brachioradialis, extensor carpi radialis longus, and extensor pollicis brevis. 3. Reduced in size, plus fatty changes :-deltoid, brachialis, and radial head of abductor pollicis longus.

When the muscles were dissected off the range of movement of the joint was increased by 5 degrees. The lower end of the humerus was straight, and from the front presented a fulness instead of the usual radial and coronoid fossæ. The radial collateral ligament was strong and well defined. A strong band of fibrous tissue extended from the lateral epicondyle of the humerus to the posterior border of the ulna below the lesser sigmoid notch. This band was tightened on flexion and 
appeared to limit this movement. The ulnar collateral ligament was very strong. The posterior portion, normally triangular, was quadrilateral, the upper margin being attached to the medial epicondyle and the posterior part of the shaft of the humerus.

The exposed portion of the capsule appeared normal. When the joint cavity was opened the articular surfaces on the lower end of the humerus were found to be abnormal (Fig. 4). The trochlea and capitulum were confined to the inferior surface and did not extend, as in the normal, on to the front of the bone. On the

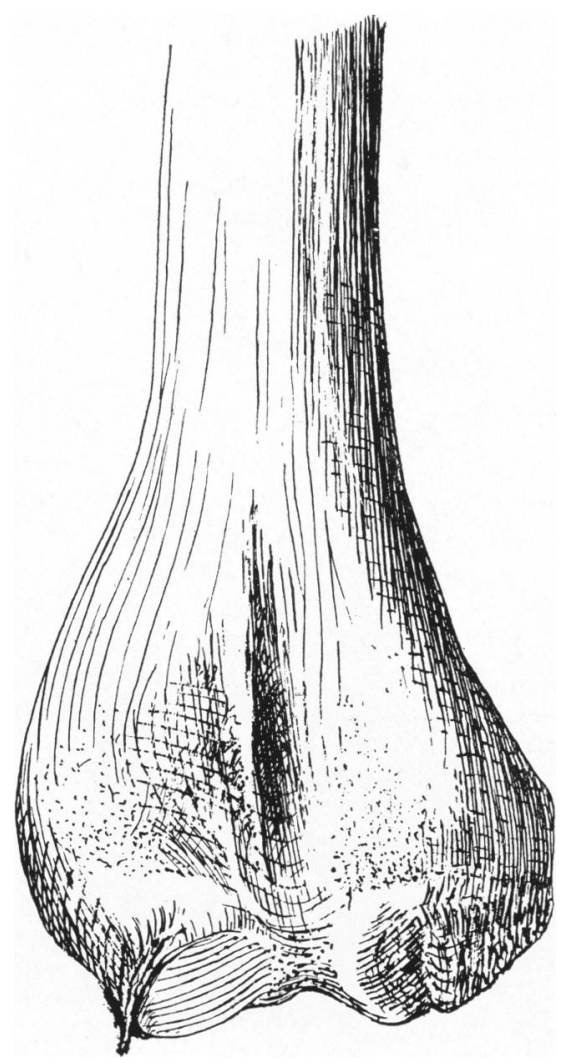

FI't. 4. Anterior aspect of lower end of humerus. Note that capitulum and trochlea do not extend on to the anterior aspect of the bone. The bone is full and convex where normally the radial and coronoid fossæ are situated.

upper part of the greater sigmoid notch, that formed by the olecranon, the articular cartilage was replaced by fibrous tissue which was attached to the olecranon fossa just above the margin of the trochlea. When this band was cut the joint could be fully flexed.

In the wrist joint the interosseous ligament between the proximal ends of the lunate and triquetrum was attached to the articular surface of the radius thus separating the radio-lunate from the radio-triquetral portion. The shoulder joint appeared normal.

With regard to the question whether the disease is primary in the joints or in the muscles it can only be remarked that if the condition in the joint is primary it is very difficult to account for the distribution of the changes in the muscles. 
Report on microscopic sections.-The thymus, spleen, kidney, and pituitary and pineal glands were normal in appearance. The muscle sections were stained with hæmatoxylin and eosin, and with Heidenhain Azan stain. The quadriceps extensor and glutæus maximus showed some degree of degeneration of muscle fibre. The flexors of the arm showed the same degenerative changes, but more widespread. The brachialis was the most degenerated, biceps next, and deltoid least. The muscle sections showed striated muscle fibres, areas of fibrous tissue, and fat replacement. There were also some infrequent zones of small atrophied fibres and chains of nuclei with degenerated sheaths. No myoblasts were seen in these sections (Fig. 5).

Report on spinal cord (Dr. R. J. Gladstone).-The specimens stained with hæmatoxylin and eosin, and by van Gieson's method, showed considerable shrinkage of the nerve cells. Both grey and white matter were feebly stained with eosin and

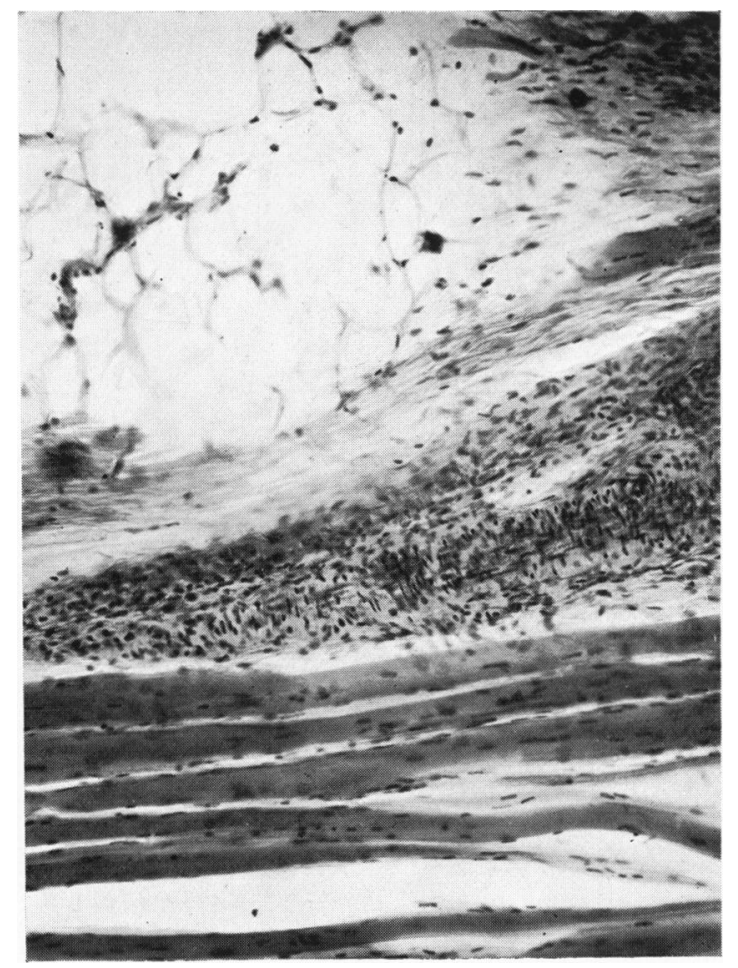

Fra. j. Longitudinal section of the deltoid showing striated fibres, atrophied tibres and fat $(\times 120)$.

the nuclei were unstained with the hæmatoxylin. The ependymal lining of the central canal of the spinal cord was complete and well stained. The canal was slightly larger than normal, the diameter being $0.12 \mathrm{~mm}$. A large U-shaped space was present in the central part of the grey matter; the bend of the $U$ was in the anterior grey commissure and the two limbs passed dorsally one on each side of the central canal. Sections of several small vessels were seen in the space and appeared to lie free in the cavity, the surrounding and supporting and nerve tissues having disappeared. Other smaller spaces and fissures were present both in the grey and white matter.

Small yellow and black particles were scattered sparsely through the section. These were present in both grey and white matter, and also in sections of the ventral 
and dorsal roots of the spinal nerves which lay in the subarachnoid space around the cord. The yellow particles appeared to be degenerate red blood corpuscles.

The multipolar ganglion cells in both the anterior and posterior cornua of the grey matter were shrunken and degenerate (Fig. 6). The cells of the anterior cornua were less affected on one side than the other. The nuclei of these cells were either unstained or feebly stained with the hæmatoxylin.

The white columns as a whole appeared degenerate, the outlines of the nerve fibres being almost indistinguishable. The tissue consisted chiefly of a fine neuroglial network traversed by blood vessels which appeared to radiate from the grey matter, and, owing to the degenerate condition of the nerve fibres, were unusually distinct. Owing to the general condition of degeneration of the nerve fibres, no specially degenerated tracts were indicated by the Marchi method; the fibres of the crossed pyramidal tract were more darkly stained by this method than the dorsal spinocerebellar tracts, and the cross section of the nerve fibres of the pyramidal tracts

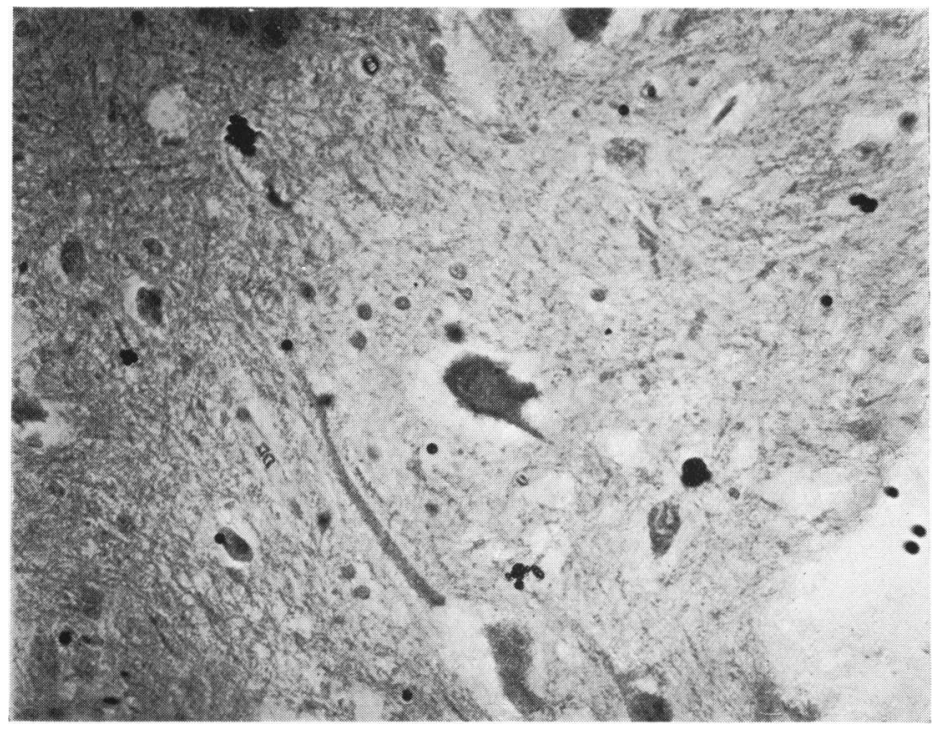

Fur. 6. Section of cond showing cells of anterior horn. Stained H. and E. $(\times 320)$.

appeared slightly more distinct than in the spino-cerebellar tracts. The antero-median columns of white matter were much distorted and reduced in the antero-posterior diameter. The grey matter of the dorsal cornua appeared to be broken up, and to consist chiefly of neuroglial fibres.

Transverse sections of the roots of spinal nerves in the subarachnoid space showed nerve fibres which for the most part were feebly stained by the Marchi method (Fig. 7). In the sections stained by van Gieson's method some nerve fibres showed a thin sheath completely stained and appearing as a black ring; in others only a part of the circumference was stained, the clear area in the centre being bounded on one side by a dark semi-circle or crescent, the remainder of the sheath being unstained.

The pia arachnoid and the dura mater were thickened, and the connective tissue fibres were well stained by both the hæmatoxylin and eosin and the van Gieson stains. The pia was very vascular. The thickening of the dura mater and increased vascularity of the pia mater appeared to be the result of a chronic inflammatory condition of the membranes. 


\section{Discussion.}

Previous writers on this condition have directed their attention mainly to the joint condition, and the disease became known as arthrogryposis multiplex congenita. Scarlini ${ }^{2}$ suggested that the cause was muscular ligamentous retraction. Stern ${ }^{3}$ regarded the condition as due to a rare combination of intra-uterine peri-arthritis and intra-uterine pressure. Rocher ${ }^{4}$ considered that compression in utero affected all tissues equally. W. Sheldon was the first to put forward the hypothesis that the muscles were primarily at fault. His investigations on the electrical reactions of the affected muscles confirmed this view. After quoting the literature and adding notes on four cases of Fairbank's besides his own, he put forward the view that the

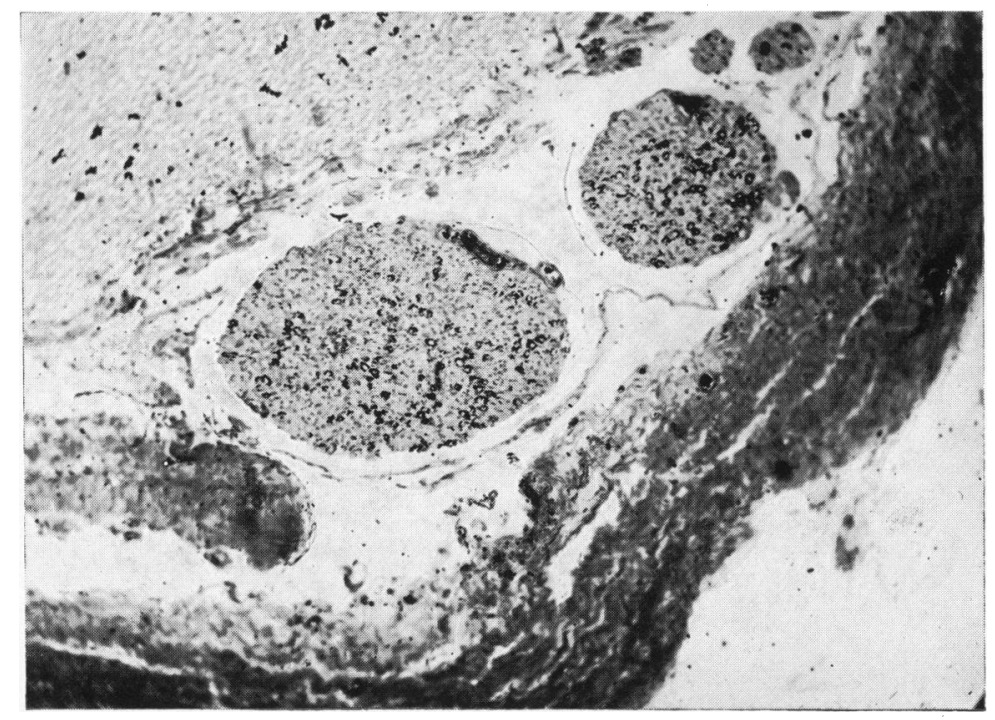

Frc. 7. Transverse section of roots of spinal nerves, showing pia, arachnoid and dura mater, in cervical region.

Stained van Gieson $(\times 100)$.

initial lesion consists of a developmental aplasia of certain muscle groups, followed by secondary developmental defects in the structure of the joints. On these grounds he advanced the name amyoplasia congenita as giving a more correct description of the condition than the names formerly used.

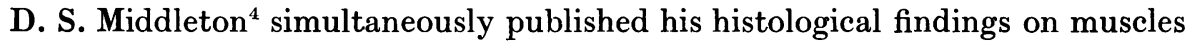
obtained by biopsy in cases of arthrogryposis, together with remarks on a lethal hereditary deformity in lambs, studied by Millar in the Institute of Animal Genetics in Edinburgh. Middleton suggested that the deformities were due to the failure of certain muscles to develop completely, with a tendency towards early degeneration, and he concluded that the muscular condition was the primary rtiological factor in the production of these deformities. Fraser Roberts ${ }^{6}$ on lambs with ' lethal muscle contracture' 


\section{AMYOPLASIA CONGENITA}

has drawn attention to the fact that hydrops amnii is very marked, that all affected lambs die at birth, and that when there are twins it is rare for both to be deformed. He stated that the condition in lambs depends on the homozygous state of an autosomal recessive factor.

In reviewing the present case with its post-mortem findings in the light of already published work, the following points emerge.

The joint changes in the dissected elbow were marked and such as could be accounted for by the fact that flexion had never been performed in utero. Besides the non-development of the full articular surfaces, certain fibrous bands and ligaments which developed round the joint also contributed towards limiting flexion. The removal of the muscles did not result in free flexion of the elbow joint, movement being still restricted. The chief obstacle was the fibrous tissue which replaced the articular cartilage on the upper part of the greater sigmoid notch. As the limitation of movement in each joint was directly proportionate to the amount of atrophy in the group of muscles responsible, it would appear probable that the joint changes are secondary to the muscle defects, and that the muscle degeneration must be due to some hitherto unexplained cause. The elbow joint may be compared with the condition in congenital dislocation of the hip where the acetabulum is not formed, and is filled with fibrous tissue, the head of the femur never having moved inside it. Why is there not some degree of malformation of the joints in cases of Oppenheim's amyotonia congenita? It may be suggested that the muscle aplasia in this condition is distributed uniformly without antagonistic grouping, leaving a flaccid joint, so that there is some voluntary and perhaps also some flail movement in utern.

In the case under consideration the changes in the affected groups of muscles were marked both naked-eye and microscopically. The muscles found to be most reduced in size on dissection showed most degeneration on section. This leads to the question whether the muscles are ætiologically absent, or whether it is an early intra-uterine degenerative process. Comparing the muscles of the infantile myopathies with this case we find that they are not unlike. In Werdnig-Hoffmann and in Oppenheim's diseases $^{7,8}$ the muscle sections show fibrous tissue, fat replacement, and in many cases great reduction in the size of fibres, which may be so atrophied that they appear to be mere chains of nuclei. The picture of the affected muscles in amyoplasia congenita shows similar degenerative changes, though not so severe, and there are considerably fewer small fibres. There seems to be reason from the appearance of the muscles to place amyoplasia congenita in the same class as the infantile myopathies.

In comparing this condition with the lethal condition in the herd of lambs in Edinburgh, several points of difference are to be found. The disease in lambs appears to be a Mendelian characteristic; this has never been shown in this condition in humans. In lambs hydrops amnii is a constant feature; in children there is frequently reccrded some amniotic disturbance, either hydramnios or oligamnios, but often there is no history of any such 
abnormality. In lambs there are no secondary joint changes, which may be partly due to the presence of hydramnios, allowing of a free flail-like movement in utero. The diaphragm is always affected in lambs, and invariably causes death at birth. No such case has been recorded in infants. The thymus enlargement is a constant feature in lambs: in the present case the thymus was found post mortem to be rather larger than normal, but this had not been observed clinically or by X-rays, and on section the gland appeared normal.

The spinal cord had been kept for some months in formalin before it was examined. The cells in the anterior horn appeared to be normal in size and number, but there was some lack of myelin in both anterior and posterior roots, with a generalized degeneration of nerve fibres. The anterior horn cells were less affected on one side than the other. In Werdnig-Hoffmann and Oppenheim's diseases, poverty of cells in the anterior horns is a constant feature: there is a diminution both in size and number, and also thinness and poor myelination of the anterior roots with signs of degeneration in the horns. From these differences in the spinal ccrd this case of amyoplasia congenita would not appear to belong to the same class as those of WerdnigHoffmann and Oppenheim. The two last menticned have not yet provided any definite signs of inflammation. These two conditions show the same pathological picture of muscle and cord, but the clinician can distinguish them from one another quite readily; they are both of the flaccid type. Amyoplasia which is of the rigid spastic type, is distinct clinically from these two, and the pathological picture of the cord is different, though the muscles show the same picture of atrophy as is commcn to the myopathies.

The sections of the cord in the case of amyoplasia showed evidence of inflammation of the meninges. Red blood corpuscles were scattered throughout the sections, the dura mater was thickened and adherent to the skull, the pia mater was vascular, the central canal of the cord was somewhat larger than normal, and the fontanelle wide. Thus we have evidence of a chronic inflammatory condition of the membranes and of a mild degree of hydrocephalus present at some stage in the developing fœtus, although clinically there were no meningeal symptoms at any time during life, nor was there any apparent hydrocephalus. We have then evidence for the conclusion that the cord changes were not secondary to the muscle condition, but that the primary fault lay within the spinal cord. The selection of groups of muscles at the level of the cervical and lumbar enlargements and the noninvolvement of all the other muscles seem to confirm the hypothesis of a central origin. The presence of inflammatory changes in the cord, and the absence of myoblasts in the muscles seem to be inconsistent with any theory of abictrophy ${ }^{9}$.

How could this central infection or injury arise, at what stage in the course of development, and why was the most injury done at the level of the VI, VII and VIII cervical segments and also in the lower lumbar region? Goodall $^{10}$ says that fœtal abnormalities such as hydrocephaly, meningocele, 
spina bifida, anencephaly, are commonly associated with hydramnios, and that they probably arise from a low grade attenuated infection of the amniotic sac. Abortion occurs early if the infection is severe. Taussig ${ }^{11}$ reported 32 cases of hydramnios, with 22 per cent. of fœtal deformities, and a mortality of 44 per cent. Krahula ${ }^{12}$ reported 72 cases of hydramnios, with $37 \cdot 5$ per cent. of fœtal deformities and a primary mortality of 54 per cent. Necrosis of the amnion is found in oligamnios. Hydramnios and oligamnios have been reported in connection with arthrogryposis sufficiently often to attract attention to the possibility of an early amniotic infection in these cases. In the embryo the amniotic fluid has access to the brain and sub-meningeal spaces until the closing of the neural tube at the fourth and fifth week of embryonic life. If the amniotic infection occurred when the neural canal had closed in all but the cervical and lower lumbar regions, one might get a deformity such as is present in amyoplasia congenita. In Oppenheim's disease such an infection, if present, would occur when the canal is still wholly open. If the infection occurs after the canal is closed the central nervous system escapes the amniotic infection. Werdnig-Hoffmann's disease is not usually observed at birth, but it may be of interest to quote a case recently under the writer's care. The mother spent Christmas day in bed with a sharp attack of influenza, and on the following 25th of August a child was born, in whom no abnormality was observed in the maternity hospital, but who died of Werdnig-Hoffmann's disease five and a half months later. Definite histories of possible sources of infection to the fœtus are not easily obtained, because at the fourth week of pregnancy the mothers have not yet begun to take note of their health. In the case of amyoplasia under consideration no such history could be elicited. It is, however, possible that, after eight pregnancies in close succession, the mother might have been suffering from a mild chronic endometritis without complaining of it. Some information of value might be gained if this question of early infection during intra-uterine life were followed up, and if in these cases a close examination were made into the history of the mother's health during the fourth and fifth weeks of pregnancy.

\section{Summary.}

1. In a case of amyoplasia post-mortem evidence is presented to show that the condition of the joints is secondary to that of the muscles.

2. The cord showed definite signs of meningeal inflammation and a slight degree of hydrocephaly.

4. The question of an amniotic infection at the fourth and fifth weeks

3. The disease is compared with Werdnig-Hoffmann's and Oppenheim's diseases. of intra-uterine life is considered.

My thanks are due to the following who have assisted in the preparation of this communication:-Dr. A. R. Halpenny for performing the 
autopsy, and for preparing the sections of the muscles and organs; Dr. C. P. Martin, University Anatomist, and Mr. McEntee, of the School of Anatomy, Trinity College, for the dissection of the arm; Mr. Middleton for examining the muscle sections and for allowing me to examine sections of lamb muscle; Dr. R. J. Gladstone, of King's College, London, for preparing and reporting on the sections of the spinal cord; Dr. Greenfield for examining the cord and muscle sections; Mr. H. A. T. Fairbank for examining the dissection of the elbow joint; and Dr. W. Sheldon for help and advice.

\section{REFERENGES.}

1. Sheldon, W., Arch. Dis. Childh., Lond., 1932, VII, 117.

2. Scarlini, G., Arch. di Orton., Milan, 1926, XLII, 451.

3. Stern, W. G., J. Am. Med. Ass., Chicago, 1923, LXXXI, 1507.

4. Rocher, H. L., J. méd. de Bordeaux, Bordeaux, 1913, LXXXIV, 772.

5. Middleton, D. S., Edinburgh Med. J., Edin., 1932, XXXIX, 389.

6. Roberts, F. A. F., J. Genetics, Lond., 1929, XXI, 57.

7. Greenfield, J. G., \& Stern, R. O., Brain, Lond., 1927, L., 652.

8. Huenekins, A. B., \& Bell, E. T., Am. J. Dis. Ch., Chicago, 1920, XX, 496.

9. Gowers, W., Lancet, Lond., 1902, i, 1003.

10. Goodall, R. J., J. Obst. \& Gyn., Manchester, 1931, XXXVIII, 847.

11. Taussig, F. J., Am. J. Obst. \& Gyn., St. Louis, 1928, XIV, 505.

12. Krahula, Monatschr f. Geburtsh. u. Gyn., Berlin, 1921, LV, 199. 